\title{
SINTOMAS PSICOLÓGICOS DO ENVELHECIMENTO MASCULINO E FATORES ASSOCIADOS
}

\author{
Leandro Quadro Corrêa ${ }^{1}$ \\ Marcelo Cozzensa da Silva² \\ Airton José Rombaldi
}

resumo

Este estudo objetivou verificar a prevalência e os fatores associados aos sintomas psicológicos do envelhecimento masculino em uma amostra representativa de homens com idade igual ou superior a 40 anos, residentes na zona urbana do município de Pelotas, Rio Grande do Sul, Brasil. Foi realizado um estudo transversal de base populacional, incluindo 421 homens que residiam na zona urbana do município. Para medir os sintomas psicológicos do envelhecimento foi utilizada a dimensão psicológica da escala AMS (The Aging Males' Symptoms Scale). Considerou-se como tendo sintomas aqueles homens que apresentaram sintomas moderados e graves. A prevalência dos sintomas psicológicos do envelhecimento masculino foi de

1 Graduado em Educação Física. Mestre em Educação Física. Professor Assistente da Universidade Federal do Rio Grande (FURG), vinculado ao Instituto de Educação. E-mail: leandroqc@hotmail.com. 2 Graduado em Educação Física. Doutor em Epidemiologia. Professor Associado da Universidade Federal de Pelotas (UFPel), vinculado ao Departamento de Desporto. E-mail: cozzensa@terra.com.br. 3 Graduado em Educação Física. Doutor em Ciências do Movimento Humano. Professor Titular da Universidade Federal de Pelotas (UFPel), vinculado ao Departamento de Desporto. E-mail: ajrombaldi@ gmail.com. 
42,3\% (IC ${ }_{95 \%}$ 37,4\%-47,1\%) e, após controle para fatores de confusão, apenas a variável autopercepção de saúde permaneceu associada ao desfecho. Concluiu-se que a prevalência de sintomas psicológicos na população masculina é elevada e afetada pela percepção de saúde. Políticas de saúde pública aliadas ao aumento de hábitos de vida saudáveis poderiam minimizar esta prevalência e proporcionar melhor qualidade de vida a homens de meia idade e idosos.

palavras-chave

Homens. Psicologia em Saúde. Saúde do Idoso. Epidemiologia.

\section{Introdução}

De acordo com a Organização Mundial da Saúde (WHO, 2012) o envelhecimento populacional é um fenômeno global inevitável e previsível, que vem ocorrendo com ritmo mais acelerado em países pobres e em desenvolvimento, sendo consequência da redução da taxa de fecundidade, da diminuição da mortalidade infantil e do aumento da expectativa de vida. Neste sentido, o envelhecimento populacional é um triunfo, sendo o resultado do desenvolvimento das sociedades e atestado irrefutável das vitórias da humanidade contra as adversidades e desafios da natureza, consequência de políticas e programas dirigidos a este grupo etário (SANTOS; SILVA, 2013).

Nessa perspectiva, o envelhecimento é um processo positivo estando associado à ideia de que o indivíduo preserva seu potencial de desenvolvimento durante todo o curso da vida, havendo um equilíbrio entre suas limitações e potencialidades, as quais podem ser otimizadas por meio de intervenções específicas (SCORALICK-LEMPKE; BARBOSA, 2012). Por outro lado, entre as limitações observadas em consequência do processo de envelhecimento, foco do presente estudo, estão um conjunto de fatores que se apresentam de três formas distintas: sintomas de ordem somática, sexual e psicológica (HEINEMAN et al., 1999; CORRÊA et al., 2010; T'SJOEN et al., 2004), sendo que, entre os homens, eles tendem a se acentuar com o avanço da idade (HEINEMAN et al., 1999; CORRÊA et al., 2010; ICHIOKA et al., 2006; AKINYEMI et al., 2008; SOUZA; GUIMARÃES, 2016; YUEN et al., 2016), podendo influenciar negativamente a saúde dos mesmos (T'SJOEN et al., 2004).

Os sintomas psicológicos do envelhecimento masculino caracterizam-se por um conjunto de fatores (irritabilidade, depressão, ansiedade, nervosismo e 
sensação de esgotamento) que, quando agregados, caracterizam a sintomatologia geral (HEINEMANN et al., 1999). A prevalência deste tipo de sintoma nas populações estudadas tem variado entre 14,6\% e 42,2\% (HEINEMAN et al., 1999; CORRÊAA et al., 2010; YUEN et al., 2016; ICHIOKA et al., 2006).

Estudos epidemiológicos têm focado na prevalência e nos fatores associados a sintomas psicológicos específicos, como a depressão e a ansiedade (GAZALLE et al., 2004; ROMBALDI et al., 2010; BYERS et al., 2010; UCHMANOWICZ; GOBBENS, 2015), mas não no conjunto dos sintomas psicológicos que se apresentam no processo de envelhecimento do homem, parecendo assim, haver lacuna no conhecimento no que diz respeito a estudos que avaliem esse desfecho, assim como os fatores associados a ele. Nesse sentido, torna-se necessária a compreensão dos fatores que podem influenciar ou estarem associados ao surgimento dos sintomas psicológicos do envelhecimento masculino, bem como apresentar a parcela da população que apresenta a sintomatologia. Assim, o presente estudo teve como objetivo verificar a prevalência e os fatores associados aos sintomas psicológicos do envelhecimento masculino em uma amostra representativa de homens com idade igual ou superior a 40 anos, residentes na zona urbana do município de Pelotas, Rio Grande do Sul.

\section{Metodologia}

Trata-se de um estudo observacional com delineamento transversal de base populacional realizado na zona urbana do município de Pelotas, no ano de 2008. Essa cidade está localizada no extremo sul do Estado do Rio Grande do Sul e possui cerca de 340 mil habitantes. De acordo com o censo de 2000 do Instituto Brasileiro de Geografia e Estatística, a cidade tinha aproximadamente $32 \%$ de sua população com idade igual ou superior a 40 anos no período da coleta de dados (IBGE, 2000).

O Instituto Brasileiro de Geografia e Estatística divide o município em 408 setores censitários urbanos os quais são ordenados no formato "espiral", do centro para os bairros (IBGE, 2000). Dos 404 setores que contêm domicílios, foram sorteados 45 para serem incluídos no estudo de modo a atender o maior número amostral calculado. Em cada setor censitário sorteado identificou-se o ponto de partida do estudo, a partir do qual foram selecionados sistematicamente os domicílios a serem visitados. Após a seleção do primeiro domicílio a ser incluído no estudo, as próximas foram selecionadas de forma sistemática, respeitando-se o intervalo estipulado de cinco domicílios, até atingirmos as 20 residências em cada setor (HALLAL et al., 2010). 
No total foram selecionadas 900 residências onde todos os homens que apresentassem idade igual ou superior a 40 anos foram inicialmente considerados elegíveis para o estudo, tendo em vista o instrumento utilizado para medir o desfecho. Foram excluídos do estudo indivíduos institucionalizados (asilos, hospitais, prisões e quartéis), indivíduos com incapacidade motora severa (tetraplégicos, paralisia cerebral, entre outras) e indivíduos que não tivessem capacidade de responder e/ou compreender ao questionário.

Optou-se pela seleção de 45 setores e 20 domicílios em cada um deles, totalizando 900 domicílios a serem visitados. Tal fato foi ocasionado por esse estudo ter feito parte de um projeto maior (consórcio de pesquisa), no qual outros desfechos em estudo necessitavam maior tamanho amostral (HALLAL et al., 2010).

Para o cálculo do tamanho amostral foi utilizado uma estimativa de prevalência de $20 \%$ de sintomas do envelhecimento masculino para homens de 40 anos ou mais, um erro aceitável de quatro pontos percentuais e nível de significância de 95\%. O tamanho amostral calculado, inicialmente, foi de 384 homens. Foi acrescido a esse valor $10 \%$ para perdas e recusas, sendo o " $n$ " final de 421 sujeitos com idade de 40 anos ou mais.

O desfecho, sintomas psicológicos do envelhecimento masculino, foi medido através de um bloco de cinco questões da The Aging Males' Symptoms Scale (AMS), validada por Heineman et al. (1999). Cada questão pode fornecer um escore de um a cinco pontos, sendo que um ponto representa a não existência de sintoma e cinco a presença mais grave. Na primeira questão deste bloco foi perguntado aos participantes sobre irritabilidade (se sentia agressivo, facilmente perturbado com pequenas coisas, com humor instável), na segunda sobre nervosismo (se sentia tensão interior, agitação, sentia-se desassossegado), na terceira sobre ansiedade (sentimento de pânico), na quarta sobre sintomas de depressão (sentia-se para baixo, triste, à beira das lágrimas, com falta de energia, alterações de humor, sensação de que nada vale a pena) e na última como estava sua sensação de esgotamento. A pontuação gerada pelas questões do instrumento classifica como não possuidores dos sintomas os sujeitos que apresentaram escores até cinco pontos; os que apresentassem entre 6 e 8 pontos como tendo sintomas fracos; entre 9 e 11 pontos com sintomas moderados; e os sujeitos que tiveram escore maior ou igual a 12 pontos com sintomas psicológicos muito graves. Para fins de análise, no entanto, o escore foi dicotomizado e foi considerado como tendo sintomas psicológicos do envelhecimento masculino aqueles homens que apresentaram sintomas de moderados a muito graves (pontuação maior ou igual a nove pontos).

Quando analisadas isoladamente as diferentes questões que compõem o escore psicológico, foram classificados como não tendo sintomas aqueles 
que apresentassem um ponto, como tendo sintomas leves e moderados os com pontuação de dois e três pontos e como tendo sintomas graves ou muito graves aqueles que apresentassem quatro ou cinco pontos. Foram considerados como tendo algum dos sintomas aqueles que apresentaram valores igual ou superior a três pontos.

Características demográficas, socioeconômicas e de saúde foram coletadas através da aplicação de um questionário padronizado pré-testado em um setor censitário que não fez parte da amostra (estudo piloto). As variáveis independentes foram idade (em anos completos), cor da pele (dividida em branca; não branca, conforme percepção do entrevistador), situação conjugal (com companheiro; sem companheiro), nível econômico - determinado segundo classificação da Associação Brasileira de Empresas de Pesquisa (ABEP, 2008) com o nível A de mais elevado, seguido por B; C; D/E), escolaridade (em anos completos de estudo), tabagismo (fumante atual; ex-fumante; nunca fumou) e autopercepção de saúde (excelente; muito boa; boa; regular; ruim). O estado nutricional foi determinado pelo índice de massa corporal (IMC), calculado pelo peso e altura referidos e classificado segundo critérios da WHO (1998). Para definir o escore de atividade física dos entrevistados, utilizou-se somente as sessões de lazer e deslocamento do International Physical Activity Questionnaire (IPAQ; CRAIG et al., 2003), tendo em vista que o nível de atividade física relatado nas seções do trabalho e doméstico parece ser superestimado (HHS, 2008). Foram considerados ativos aqueles que atingiram o mínimo de $150 \mathrm{~min} /$ semana de atividades físicas nos dois domínios analisados de forma conjunta, de acordo com as recomendações do American College of Sports Medicine e World Health Organization (HASKELL, 2007; WHO, 2010).

$\mathrm{O}$ instrumento foi aplicado face-a-face por entrevistadores de ambos os sexos com, pelo menos, ensino médio completo, que receberam treinamento de 40 horas para aplicação do instrumento sem estarem informados dos objetivos nem das hipóteses do estudo. Os entrevistadores realizaram as entrevistas individualmente. Os supervisores do trabalho de campo refizeram as entrevistas em $10 \%$ da amostra, selecionada ao acaso, por meio de um questionário reduzido, contendo questões-chave selecionadas do instrumento como controle de qualidade do estudo.

O banco de dados foi construído no programa Epi Info 6.0, sendo realizada dupla digitação de cada questionário. Para análise dos dados utilizou-se o programa STATA 14. Foi feita uma análise descritiva dos participantes da amostra em termos de sintomas psicológicos e das variáveis socioeconômicas, demográficas, comportamentais, nutricionais e de saúde. Na análise bivariada foi verificada a relação entre o desfecho e as variáveis independentes por meio 
dos testes de Qui-quadrado para heterogeneidade e tendência linear, quando as variáveis não atendiam algum dos pressupostos do teste utilizou-se o teste exato de Fisher. A análise multivariável foi conduzida através de regressão de Poisson, respeitando um modelo hierárquico composto por quatro níveis. No nível mais distal foram incluídas as variáveis idade e cor da pele; no segundo nível, escolaridade, nível econômico, trabalho fora e situação conjugal; no terceiro nível estão IMC, tabagismo e nível de atividade física no lazer e no deslocamento; e no nível proximal, os sintomas sexuais do envelhecimento masculino e a autopercepção de saúde.

Os efeitos das variáveis foram controlados para as variáveis do mesmo nível e superiores, sendo mantidas na análise todas aquelas que apresentaram valor $\mathrm{p} \leq 0,2$. Quando foram testados individualmente os desfechos que compõem a sintomatologia psicológica geral, utilizou-se regressão Logística, tendo em vista que os desfechos foram avaliados de forma categórica ordinal (sem sintoma; sintomas leves/moderados; sintomas graves/muito graves) e respeitou-se o mesmo modelo hierárquico. Todas as análises levaram em consideração o efeito do delineamento amostral, pelo grupo de comandos "svy" do Stata. O nível de significância estabelecido foi de 5\%.

Este estudo foi aprovado pelo Comitê de Ética em Pesquisa da Escola Superior de Educação Física da Universidade Federal de Pelotas (protocolo 005/2008). Os dados foram coletados após consentimento informado dos participantes.

\section{Resultados}

Participaram do estudo 421 sujeitos de 40 anos ou mais, em 876 domicílios, sendo que o total de perdas e recusas foi de $8,3 \%$. O efeito de delineamento encontrado $(0,7)$ foi suficiente para manter o poder e o nível de confiança previstos pelo estudo. Em relação ao desfecho em estudo - sintomas psicológicos do envelhecimento - foram obtidas informações de 415 participantes.

A média de idade dos homens entrevistados foi $54,5 \pm 10,5$ anos e $29,9 \%$ tinham até quatro anos de estudo. Dos entrevistados, aproximadamente 85,0\% eram de cor branca, quase metade encontrava-se no nível socioeconômico C $(46,2 \%)$ e $77,2 \%$ eram casados ou viviam com companheira. Observou-se que pouco mais de $1 / 5$ dos respondentes eram fumantes no momento da entrevista, 67,1\% apresentavam IMC correspondente à sobrepeso/obesidade, $82,9 \%$ eram sedentários nos domínios analisados e $23,8 \%$ percebiam sua saúde como regular ou ruim (Tabela 1). A prevalência dos sintomas psicológicos do envelhecimento masculino foi de $42,3 \%$ ( $\left.\mathrm{IC}_{95 \%} 37,4-47,1\right)$. Quando os sintomas 
que compõem a sintomatologia psicológica geral do envelhecimento masculino foram analisados separadamente, identificou-se prevalências de 16,4\% ( $\left.\mathrm{IC}_{95 \%} 12,9-20,3\right)$ para a irritabilidade, $16,1 \%\left(\mathrm{IC}_{95 \%} 12,7-20,0\right)$ para o nervosismo, $21,7 \%\left(\mathrm{IC}_{95 \%} 17,8-25,9\right)$ para a ansiedade, $11,6 \%\left(\mathrm{IC}_{95 \%} 8,7-15,0\right)$ para a depressão e de $49,6 \%\left(\mathrm{IC}_{95 \%} 44,7-54,6\right)$ para o esgotamento.

Tabela 1 - Descrição da amostra de acordo com as variáveis independentes em estudo $(n=421)$.

\begin{tabular}{|c|c|c|}
\hline Variáveis & $\mathrm{N}$ & $\%$ \\
\hline \multicolumn{3}{|l|}{ Idade em anos completos } \\
\hline $40-49$ & 161 & 38,8 \\
\hline $50-59$ & 134 & 32,3 \\
\hline $60-69$ & 77 & 18,6 \\
\hline 70 ou mais & 43 & 10,3 \\
\hline \multicolumn{3}{|l|}{ Cor da pele } \\
\hline Branca & 334 & 86,1 \\
\hline Não branca & 54 & 13,9 \\
\hline \multicolumn{3}{|c|}{ Escolaridade em anos completos de estudo } \\
\hline 0 & 13 & 3,3 \\
\hline 1 a 4 & 100 & 25,6 \\
\hline 5 a 8 & 135 & 34,6 \\
\hline 9 a 11 & 72 & 18,5 \\
\hline 12 ou mais & 70 & 18,0 \\
\hline \multicolumn{3}{|l|}{ Nível socioeconômico } \\
\hline A (mais elevado) & 31 & 8,1 \\
\hline B & 129 & 33,6 \\
\hline C & 178 & 46,4 \\
\hline D/E (mais baixo) & 46 & 11,9 \\
\hline \multicolumn{3}{|l|}{ Trabalho fora } \\
\hline Não & 141 & 33,5 \\
\hline Sim & 280 & 66,5 \\
\hline \multicolumn{3}{|l|}{ Situação conjugal } \\
\hline Casado ou morando com companheira & 304 & 77,9 \\
\hline Sem companheira & 86 & 22,1 \\
\hline \multicolumn{3}{|l|}{ IMC (Kg/min²) } \\
\hline Normal & 123 & 32,9 \\
\hline Sobrepeso & 171 & 45,7 \\
\hline
\end{tabular}


continuação

\begin{tabular}{l|c|c}
\hline Variáveis & N & $\%$ \\
\hline Obesidade & 80 & 21,4 \\
\hline Tabagismo & & \\
\hline Nunca fumou & 116 & 29,7 \\
\hline Ex-fumante & 165 & 42,3 \\
\hline Fumante atual & 109 & 28,0 \\
\hline $\begin{array}{l}\text { Nível de atividade física de lazer e } \\
\text { deslocamento }\end{array}$ & & \\
\hline Sedentários & 349 & 82,9 \\
\hline Ativos & 72 & 17,1 \\
\hline Autopercepção de saúde & 37 & 9,5 \\
\hline Excelente & 54 & 13,8 \\
\hline Muito boa & 205 & 52,6 \\
\hline Boa & 74 & 19,0 \\
\hline Regular & 19 & 4,8 \\
\hline Ruim & & \\
\hline Sintomas psicológicos moderados/severos & 240 & 57,8 \\
\hline Não & 175 & 42,8 \\
\hline Sim & & \\
\hline
\end{tabular}

IMC = Índice de Massa Corporal.

Fonte: elaborado pelos autores.

Na análise bivariada (Tabela 2), observou-se que os sintomas psicológicos do envelhecimento masculino estiveram significativamente e inversamente associados com a pior percepção de saúde, associação que se manteve na análise multivariável após ajuste para os fatores de confusão (Tabela 3). Aqueles que perceberam sua saúde como ruim tiveram 2,1 vezes mais chance que os que percebiam sua saúde como excelente de apresentar sintomas psicológicos do envelhecimento (Tabela 3). 
Tabela 2 - Prevalência de sintomas psicológicos do envelhecimento masculino e associação bivariada entre sintomas sexuais e variáveis independentes em estudo ( $n=421$ ).

\begin{tabular}{|c|c|c|c|c|}
\hline \multirow[b]{2}{*}{ Variáveis } & \multirow[b]{2}{*}{ N } & \multirow[b]{2}{*}{$\%$} & \multicolumn{2}{|c|}{ Análise bruta } \\
\hline & & & $\mathrm{RP}(\mathrm{IC} 95 \%)$ & Valor $\mathrm{p}$ \\
\hline Idade (anos completos) & & & & $<0,6^{\star \star}$ \\
\hline $40-49$ & 69 & 42,9 & 1,0 & \\
\hline $50-59$ & 60 & 44,8 & $1,0(0,8$ a 1,4$)$ & \\
\hline $60-69$ & 28 & 36,4 & $0,8(0,6$ a 1,2$)$ & \\
\hline 70 ou mais & 18 & 41,9 & $1,0(0,6$ a 1,5$)$ & \\
\hline Cor da pele & & & & $0,7^{\star}$ \\
\hline Branca & 149 & 42,3 & 1,0 & \\
\hline Não branca & 24 & 39,3 & $0,9(0,6$ a 1,4$)$ & \\
\hline $\begin{array}{l}\text { Escolaridade } \\
\text { (anos completos de estudo) }\end{array}$ & & & & $0,4^{* *}$ \\
\hline 0 & 7 & 50,0 & 1,0 & \\
\hline 1 a 4 & 53 & 47,8 & $1,0(0,5$ a 1,8$)$ & \\
\hline 5 a 8 & 57 & 39,3 & $0,8(0,4$ a 1,5$)$ & \\
\hline 9 a 11 & 34 & 45,3 & $0,9(0,5$ a 1,7$)$ & \\
\hline 12 ou mais & 24 & 34,3 & $0,7(0,3$ a 1,4$)$ & \\
\hline Nível socioeconômico (ABEP) & & & & $0,7^{* *}$ \\
\hline A (mais elevado) & 9 & 28,1 & 1,0 & \\
\hline B & 57 & 41,6 & $1,5(0,7$ a 3,1$)$ & \\
\hline C & 83 & 43,5 & $1,5(0,8$ a 3,1$)$ & \\
\hline $\mathrm{D} / \mathrm{E}$ & 20 & 42,6 & $1,5(0,7$ a 3,2$)$ & \\
\hline Trabalho fora & & & & $0,4^{*}$ \\
\hline Não & 62 & 45,3 & 1,0 & \\
\hline Sim & 113 & 40,7 & $0,9(0,7$ a 1,1$)$ & \\
\hline Situação conjugal & & & & $0,4^{*}$ \\
\hline $\begin{array}{l}\text { Casado ou morando com } \\
\text { companheira }\end{array}$ & 131 & 40,8 & 1,0 & \\
\hline Sem companheira & 44 & 46,8 & $1,1(0,8$ a 1,6$)$ & \\
\hline IMC (Kg/min²) & & & & $0,5^{\star *}$ \\
\hline Normal & 54 & 41,2 & 1,0 & \\
\hline Sobrepeso & 71 & 39,7 & $1,0(0,7$ a 1,3$)$ & \\
\hline Obesidade & 42 & 47,7 & $1,2(0,8$ a 1,6$)$ & \\
\hline
\end{tabular}

continua 
continuação

\begin{tabular}{|c|c|c|c|c|}
\hline & & & \multicolumn{2}{|c|}{ Análise bruta } \\
\hline Tabagismo & & & & $0,2^{*}$ \\
\hline Nunca fumou & 44 & 35,2 & 1,0 & \\
\hline Ex-fumante & 74 & 43,0 & $1,2(0,9$ a 1,6$)$ & \\
\hline Fumante atual & 57 & 48,3 & $1,4(1,0$ a 1,9$)$ & \\
\hline $\begin{array}{l}\text { Nível de atividade física no lazer } \\
\text { e deslocamento }\end{array}$ & & & & $0,08^{*}$ \\
\hline Ativo & 22 & 31 & 1,0 & \\
\hline Sedentário & 154 & 44,5 & $1,4(1,0$ a 2,2) & \\
\hline Autopercepção de saúde & & & & $<0,001^{\star *}$ \\
\hline Excelente & 13 & 32,5 & 1,0 & \\
\hline Muito boa & 17 & 29,8 & $0,9(0,5$ a 1,7$)$ & \\
\hline Boa & 82 & 38,5 & $1,2(0,7$ a 1,9$)$ & \\
\hline Regular & 47 & 56,0 & $1,7(1,1$ a 2,8$)$ & \\
\hline Ruim & 15 & 75,0 & $2,3(1,4$ a 3,8$)$ & \\
\hline
\end{tabular}

* Qui-quadrado para heterogeneidade; ${ }^{* \star}$ Qui-quadrado para tendência linear.

Fonte: elaborado pelos autores.

Tabela 3 - Análise multivariável da associação entre os sintomas psicológicos do envelhecimento masculino e variáveis independentes em estudo $(n=421)^{\#}$.

\begin{tabular}{l|c|c}
\hline & \multicolumn{2}{|c}{ Análise ajustada } \\
\hline Variáveis & RP (IC 95\%) & Valor $\mathbf{p}$ \\
\hline Tabagismo & 1,0 & \\
\hline Nunca fumou & $1,1(0,9$ a 1,5$)$ & \\
\hline Ex-fumante & $1,3(0,9$ a 1,8$)$ & $0,3^{*}$ \\
\hline Fumante atual & & \\
\hline $\begin{array}{l}\text { Nível de atividade física no lazer } \\
\text { e deslocamento }\end{array}$ & $1,2(0,8$ a 1,8$)$ & \\
\hline Ativo & & $<0,001^{* *}$ \\
\hline Sedentário & 1,0 & \\
\hline Autopercepção de saúde & $0,9(0,5$ a 1,7$)$ & \\
\hline Excelente & $1,1(0,7$ a 1,8$)$ & \\
\hline Muito boa & $1,6(1,0$ a 2,5$)$ & \\
\hline Boa & $2,1(1,3$ a 3,3$)$ & \\
\hline Regular & & \\
\hline Ruim & & \\
\hline
\end{tabular}

\# Foram mantidas na análise multivariável todas variáveis que na análise bivariada apresentaram valor $\mathrm{p} \leq 0,2$; * Teste de Wald para heterogeneidade; ** Teste de Wald para tendência linear.

Fonte: elaborado pelos autores. 
A associação da variável autopercepção de saúde também foi testada com os diferentes tipos de sintomas que compõem o escore geral dos sintomas psicológicos do envelhecimento masculino. Observou-se uma associação inversa entre a pior percepção de saúde com os sintomas de irritabilidade ( $\mathrm{p}$ $<0,001)$, nervosismo $(\mathrm{p}<0,001)$, ansiedade $(\mathrm{p}=0,006)$, depressão $(\mathrm{p}<0,001)$ e esgotamento $(\mathrm{p}<0,001)$. Após análise multivariável, verificou-se que a percepção de saúde se manteve associada inversamente aos sintomas de nervosismo ( $p=0,001 ;$ RO 1,6; IC 95\% 1,2 a 2,1), ansiedade ( $p=0,001 ;$ RO 1,4; IC95\% 1,2 a 1,8) e depressão ( $p<0,001$; RO 1,7; IC 95\% 1,3 a 2,2).

A Figura 1 apresenta a prevalência dos diferentes tipos de sintomas que compõem a sintomatologia psicológica geral do envelhecimento masculino. A sensação de esgotamento foi a mais relatada (59,1\% relataram sintomas leves/moderados e 7,2\% relataram sintomas graves/muito graves), seguida da sensação de ansiedade (45,8\% reportaram sintomas leves/moderados e 5,3\% graves/muito graves).

Figura 1 - Frequência dos diferentes sintomas psicológicos relacionados aos sintomas gerais do envelhecimento masculino, necessários para a compreensão da sintomatologia geral da amostra estudada.

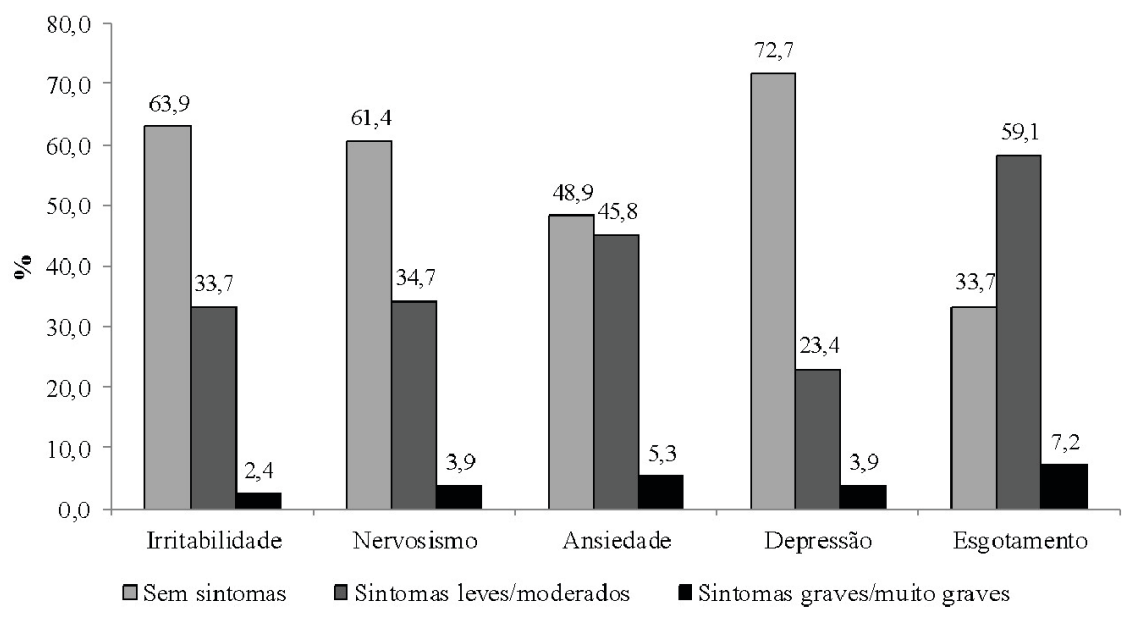

Fonte: elaborado pelos autores. 
Este estudo visou verificar os fatores que se associaram com sintomas psicológicos do envelhecimento em uma amostra representativa de homens com idade igual ou superior a 40 anos de idade residentes na zona urbana da cidade de Pelotas/RS. Um aspecto a ser destacado é que a amostra estudada é representativa dos adultos de 40 anos ou mais de idade residentes na zona urbana da cidade de Pelotas, tendo em vista o processo de seleção amostral realizado e o baixo índice de perdas e recusas. Os principais achados deste estudo foram a associação dos sintomas psicológicos do envelhecimento com a autopercepção de saúde nos homens estudados, bem como a associação entre a autopercepção de saúde com os sintomas de nervosismo, ansiedade e depressão, desfechos que compõem a sintomatologia geral do envelhecimento psicológico masculino.

A prevalência do desfecho encontrada foi de 42,3\%, significativamente maior que a descrita em outros estudos internacionais (HEINEMANN et al., 1999; ICHIOKA et al., 2006), entretanto é necessário ter cuidado quando se compara populações diferentes pois os níveis socioeconômico e educacional certamente influenciam no processo de envelhecimento de forma distinta em cada país. Além disto, o estudo de Ichioka et al., (2006) foi realizado com um grupo de homens que frequentavam um serviço de saúde no Japão, deste modo podendo ter havido influência positiva da socialização para redução deste tipo de sintomas e mudanças de comportamento. Por outro lado, a prevalência foi menor que a encontrada em estudo realizado na Nigéria (AKINYEMI et al., 2008), onde a frequência de sintomas psicológicos moderados e severos foi de $23,5 \%$ e $47,8 \%$, respectivamente; no entanto, os indivíduos considerados naquele estudo tinham idade igual ou superior a 60 anos, enquanto no presente estudo apenas 29,0\% da amostra encontravam-se nesta faixa etária. Quando se verificou os resultados do grupo etário semelhante ao do trabalho nigeriano, identificou-se prevalências dos sintomas psicológicos em 36,4\% daqueles com idade entre 60 e 69 anos e 41,9\% naqueles com 70 anos ou mais.

Em relação aos diferentes tipos de sintomas que compõem o quadro geral dos sintomas psicológicos do envelhecimento, percebeu-se elevada prevalência dos mesmos, sendo que os sintomas de esgotamento foram os mais relatados, seguido pelos sintomas de ansiedade. Esses resultados diferem do encontrado em estudo realizado na Itália (PARAZZINI; CHIAFFARINO; RICCI, 2006), onde a irritabilidade foi o sintoma que apresentou maior frequência $(23,2 \%)$ seguido dos sintomas de nervosismo relatados por $21,2 \%$ da amostra. Cabe destacar que nesse estudo italiano, a prevalência dos sintomas psicológicos gerais foi inferior aos aqui apresentados, sendo que $21,7 \%$ da amostra relatou 
ter sintomas moderados e 13,7 \% relatou sintomas severos. Entretanto os critérios de seleção dos indivíduos deste estudo foram diferentes dos utilizados aqui, tendo em vista que os homens participantes do estudo foram selecionados através do registro em clínicas gerais de cinco áreas da Itália, Desio e Palmanova/Gorizia na região norte, Parma e Firenzi na região central e Napoli na região sul, enquanto aqui foram selecionados apenas homens da área central do município de Pelotas, extremo sul do Rio Grande do Sul, o que pode ter implicado em diferenças culturais e comportamentais relevantes e consequentemente diferença nos resultados.

Em relação aos fatores associados ao desfecho, verificou-se que a única variável associada ao desfecho na análise multivariável foi a autopercepção de saúde, sendo que os homens que perceberam sua saúde como ruim tiveram risco aumentado em 110,0\% de apresentar sintomas psicológicos do envelhecimento em comparação com aqueles que perceberam sua saúde como excelente. Em estudo realizado no Japão, Hirokawa e colaboradores (2015) verificaram em 192 trabalhadores (52,2 $\pm 7,6$ anos) que os sintomas psicológicos do envelhecimento estiveram associados a redução nos níveis de testosterona e ao maior nível de estresse no trabalho. Apesar de o presente estudo não ter avaliado tais desfechos, cabe ressaltar que $66,5 \%$ dos homens aqui avaliados relataram trabalhar fora de casa, sendo que esta variável não apresentou qualquer associação com os sintomas psicológicos.

Por outro lado, Corrêa et al. (2010) relataram que a autopercepção de saúde se associou de forma linear aos sintomas gerais do envelhecimento masculino. Geralmente aquelas pessoas que percebem sua saúde como ruim apresentam, realmente, algum tipo de problema, sendo que estudos têm demonstrado associação desta variável com a indicação de mortalidade precoce e com a presença de um maior número de doenças crônicas em homens que percebem sua saúde como ruim (MCGEE et al., 1999; ALVES; RODRIGUES, 2005; ASFAR et al., 2007; JUSTO et al., 2010; DESALVO et al., 2006). Além disto, conforme Idler (2003), a autopercepção de saúde pode apresentar maior sensibilidade em homens quando comparados às mulheres, pois quando estes percebem sua saúde de forma negativa, o risco de ocorrência de um evento fatal é maior.

Algumas limitações do presente estudo precisam ser apontadas. O fato de não ter sido investigado na amostra a presença de algumas doenças que poderiam se associar ao desfecho e a possibilidade da ocorrência de causalidade reversa, típica em delineamentos transversais (especialmente em relação as variáveis nível de atividade física e autopercepção de saúde) são fatores a serem considerados. Como pontos positivos, pode-se destacar: a existência de poucos estudos avaliando os sintomas psicológicos do envelhecimento e sua 
associação com desfechos de saúde, o delineamento bem controlado, a minuciosa condução do trabalho de campo e o baixo índice de perdas e recusas em participar da pesquisa.

\section{Considerações Finais}

A prevalência de sintomas psicológicos na população masculina com 40 anos ou mais foi elevada e mostrou-se associada a autopercepção de saúde. Políticas de saúde pública aliada a hábitos de vida saudáveis podem minimizar esta prevalência e proporcionar melhor qualidade de vida a pessoas nessa faixa etária. Sugere-se a realização de novos estudos, com delineamento longitudinal, que busquem identificar possíveis fatores que influenciam no processo de envelhecimento os quais não foram abordados no presente estudo, tais como relações sociais, uso de medicamentos e doenças concorrentes.

\section{PSYCHOLOGICAL SYMPTOMS TO AGING MALES AND ASSOCIATED FACTORS}

abstract

This study aimed to identify the prevalence and associated factors from psychological symptoms of aging males in a representative sample of men with 40 years or older living in the urban area of Pelotas, Rio Grande do Sul, Brazil. A population-based cross-sectional study including 421 men living in urban area. To evaluate the psychological symptoms of aging males was used the psychological dimension of the AMS scale (The Aging Males Symptoms Scale). It was considered as having psychological symptoms men who had moderate to severe symptoms. The prevalence of psychological symptoms of aging males was $42.3 \%\left(\mathrm{Cl}_{95 \%} 37.4 \%\right.$ - 47.1\%), and after controlling for confounding factors health self-rated remained associated with the outcome. We conclude that the prevalence of psychological symptoms in older males is high, and affects their perception of health. Public health policies coupled with increased healthy lifestyle habits could minimize the prevalence, and provide better quality of life for middle-age and older men.

keywords

Men. Behavioral Medicine. Health of the Elderly. Epidemiology. 
AKINYEMI, Akanni et al. The Nigerian Aging Males' Symptoms scale. Experience in elderly males. The Aging Male, United Kingdom, v. 11, n. 2, p. 89-93, jun. 2008. Disponível em: <http://www.tandfonline.com/doi/abs/10.1080/13685530802169871?jou rnalCode=itam20>. Acesso em: 4 out. 2015.

ALVES, Luciana Correia; RODRIGUES, Roberto Nascimento. Determinantes da autopercepcão de saúde entre idosos do Município de São Paulo, Brasil. Revista Panamericana de Salud Publica, Washington, v. 17, n. 5/6, p. 333-341, maio/jun. 2005. Disponível em: <http://www.scielosp.org/pdf/rpsp/v17n5-6/26270.pdf>. Acesso em: 23 out. 2015.

ASFAR, Taghrid et al. Self-rated health and its determinants among adults in Syria: a model from the Middle East. BMC Public Health, United Kingdon, v. 7, n. 177, p. 1-9, jul. 2007. Disponível em: <http://bmcpublichealth.biomedcentral.com/articles/10.1186/1471-2458-7-177>. Acesso em: 24 out. 2015.

ASSOCIAÇÃO BRASILEIRA DE EMPRESAS DE PESQUISA (ABEP). Critério de Classificação Econômica Brasil. Disponível em: <www.abep.org>. Acesso em: 20 set. 2014.

BYERS, Amy L. High Occurrence of Mood and Anxiety Disorders among Older Adults: The National Comorbidity Survey Replication. Archives of General Psychiatry, Chicago, v. 67, n. 5, p. 489-496, maio 2010. Disponível em: <http://www.ncbi.nlm.nih.gov/pmc/ articles/PMC2933177/>. Acesso em: 20 abr. 2016.

CORRÊA, Leandro Quadro et al. Aging male's symptoms in a Southern Brazil population: lifestyle effects after the age of 40. The Aging Male, United Kingdom, v. 13, n. 2, p. 93-99, jun. 2010. Disponível em: <http://www.tandfonline.com/doi/ abs/10.3109/13685530903431597>. Acesso em: 9 out. 2014.

CRAIG, Cora L. et al. International physical activity questionnaire: 12-country reliability and validity. Medicine \& Science in Sports \& Exercise, Indianapolis, v. 35, p. 1381-1395, ago. 2003. Disponível em: <https://www.ncbi.nlm.nih.gov/pubmed/12900694>. Acesso em: 6 fev. 2018.

DeSALVO, Karen B. et al. Mortality prediction with a single general self-rated health question: a meta-analysis. Journal of General Internal Medicine, Bethesda, v. 21, p. 267-275, mar. 2006. Disponível em: <http://www.ncbi.nlm.nih.gov/pmc/articles/ PMC1828094/pdf/jgi0021-0267.pdf>. Acesso em: 24 out. 2015.

GAZALLE, Fernando Kratz et al. Depressive symptoms and associated factors in an elderly population in southern Brazil. Revista de Saúde Pública, São Paulo, v. 38, n. 3, p. 365-371, jun. 2004. Disponível em: <http://www.scielo.br/pdf/rsp/v38n3/20652. pdf>. Acesso em: 6 fev. 2018.

HALLAL, Pedro Curi et al. Consórcio de pesquisa: relato de uma experiência metodológica na linha de pesquisa em atividade física, nutrição e saúde do curso de mestrado em educação física da UFPel. Revista Brasileira de Atividade Física e Saúde, Pelotas, v. 14, n. 3, p. 156-163, set. 2009. Disponível em: <https://periodicos.ufpel.edu.br/ojs2/ index.php/RBAFS/article/view/769/778>. Acesso em: 23 set. 2015.

HASKELL, William L. et al. Physical activity and public health: updated recommendation for adults from the American College of Sports Medicine and the American Heart Association. Medicine \& Science in Sports \& Exercise, Indianapolis, v. 39, n. 8, p. 1423-1434, ago. 2007. Disponivel em: <http://journals.lww.com/acsm-msse/Fulltext/2007/08000/ Physical_Activity_and_Public_Health__Updated.27.aspx>. Acesso em: 25 out. 2015.

HEINEMANN, Lothar et al. A new "aging male's symptoms" (AMS) rating scale. The Aging Male, United Kingdom, v. 2, p. 105-114, jun. 1999. Disponível em: <http://www. tandfonline.com/doi/pdf/10.3109/13685539909003173>. Acesso em: 9 out. 2014. 
HIROKAWA, Kumi et al. Modification effects of changes in job demands on associations between changes in testosterone levels and andropause symptoms: 2 year follow-up study in male middle-aged japanese workers. International Journal of Behavavioral Medicine, dez. 2015. Disponível em: <http://link.springer.com/article/10.1007\%2Fs12529015-9530-y>. Acesso em: 23 mar. 2016.

ICHIOKA, Kentaro et al. Aging male's symptoms scale in Japanese men attending a multiphasic health screening clinic. Urology, Cleveland, v. 67, n. 3, p. 589-593, fev. 2006. Disponível em: <http://www.goldjournal.net/article/S0090-4295(05)01412-3/ pdf>. Acesso em: 4 out. 2015

IDLER, Elen L. Discussion: gender differences in self-rated health, in mortality, and in the relationship between the two. Gerontologist, Oxford, v. 43, p. 372-375, 2003. Disponível em: <http://gerontologist.oxfordjournals.org/content/43/3/372.full.pdf+html>. Acesso em: 24 out. 2015

INSTITUTO BRASILEIRO DE GEOGRAFIA E ESTATÍSTICA (IBGE). Censo Demográfico. 2000. Disponível em: <www.ibge.gov.br>. Acesso em: 10 out. 2014.

JUSTO, Dan et al. Sexual activity and erectile dysfunction in elderly men with angiographically documented coronary artery disease. International Journal of Impotence Research, v. 22, n. 1, p. 40-44, jan./fev. 2010. Disponivel em: <http://www.nature.com/ ijir/journal/v22/n1/pdf/ijir200945a.pdf>. Acesso em: 24 out. 2015.

McGEE, Daniel L. et al. Self-reported health status and mortality in a multiethnic US cohort. American Journal of Epidemiology, Oxford, v. 149, n. 1, p. 41-46, jan. 1999. Disponível em: <http://aje.oxfordjournals.org/content/149/1/41.long>. Acesso em: 23 out. 2015.

PARAZZINI, Fabio; CHIAFFARINO, Francesca; RICCI, Elena. The aging males' symptoms in the Italian population: results from a cross sectional study using the AMS scale. Archivio Italiano di Urologia e Andrologia, v. 78, n. 3, p. 87-91, set. 2006. Disponível em: <http://www.ncbi.nlm.nih.gov/pubmed/17137021>. Acesso em: 10 out. 2015.

ROMBALDI, Airton José et al. Prevalence of depressive symptons and associated factors among southern Brazilian adults: cross-sectional population-based study. Revista Brasileira de Epidemiologia, São Paulo, v. 13, n. 4, dez. 2010. Disponível em: <http://www.scielo.br/scielo.php?script=sci_arttext\&pid=\$1415-790X2010000400007> Acesso em: 24 mar. 2016.

SANTOS, Nayane Formiga; SILVA, Maria do Rosário de Fátima. As políticas públicas voltadas ao idoso: melhoria da qualidade de vida ou reprivatização da velhice. Revista FSA, Teresina, v. 10, n. 2, p. 358-371, abr./jun. 2013. Disponível em: <http://www4. fsanet.com.br/revista/index.php/fsa/article/viewFile/130/97>. Acesso em: 8 nov. 2016.

SCORALICK-LEMPKE, Natália Nunes; BARBOSA, Altemir José Gonçalves. Education and aging: Life-Span perspective contributions. Estudos de Psicologia, Campinas, v. 29, p. 647s-655s, out./dez. 2012. Suplemento. Disponível em: <http://www.scielo. br/pdf/estpsi/v29s1/01.pdf>. Acesso em: 8 nov. 2016.

SOUZA, Melissa de Carvalho; GUIMARÃES, Adriana Coutinho de Azevedo. Aging male symptoms: the relationship between physical activity and quality of life. Motriz: Rev. Educ. Fis., Rio Claro, v. 22, n. 2, p. 51-60, abr./jun. 2016. Disponível em: <http:// www.scielo.br/scielo.php?script=sci_arttext\&pid=\$1980-65742016000200051>. Acesso em: 8 nov. 2016.

T'SJOEN, Guy et al. Perception of males' aging symptoms, health and well-being in elderly community-dwelling men is not related to circulating androgen levels. Psychoneuroendocrinology, v. 29, n. 2, p. 201-214, fev. 2004. Disponível em: <http://www. psyneuen-journal.com/article/S0306-4530(03)00023-4/pdf>. Acesso em: 15 set. 2015.

UCHMANOWICZ, Izabella; GOBBENS, Robbert J. The relationship between frailty, anxiety and depression, and health-related quality of life in elderly patients with heart failure. Clinicals Interventions in Aging, New Zealand, v. 5, n. 10, p. 1595-1600, 
out. 2015. Disponível em: <http://www.ncbi.nlm.nih.gov/pmc/articles/PMC4599570/>. Acesso em: 20 abr. 2016.

US DEPARTMENT OF HEALTH AND HUMAN SERVICE (HSS). Physical Activity Guidelines for Americans, 2008. Disponível em: <http://www.health.gov/paguidelines/pdf/ paguide.pdf>. Acesso em: 7 set. 2015.

WORLD HEALTHORGANIZATION (WHO). Global Recommendations on physical activity for health. World Health Organization: Geneva, 2010. Disponivel em: <http://whqlibdoc. who.int/publications/2010/9789241599979_eng.pdf>. Acesso em: 15 mar. 2014

Good health adds life to years: Global brief for World Health Day 2012. Genebra: World Health Organization, 2012. Disponível em: <http://whqlibdoc.who.int/hq/2012/ WHO_DCO_WHD_2012.2_eng.pdf>. Acesso em: 25 set. 2015.

Obesity: preventing and managing the global epidemic, 1998. Disponível em: <http://apps.who.int/iris/handle/10665/42330>. Acesso em: 20 set. 2014.

YUEN, John W. et al. Aging males' symptoms and general health of adult males: a cross-sectional study. The Aging Male, United Kingdom, v. 19, n. 2, p. 71-78, jun. 2016. Disponível em: <http://www.tandfonline.com/doi/abs/10.3109/13685538.2016.11481 30?journalCode=itam20>. Acesso em: 8 nov. 2016.

Data de submissão: 21/04/2016

Data de aprovação: 06/04/2017 
